\title{
IncRNA AK141955 Acts as a ceRNA to Regulate Macrophage Polarization via the miR-124- 3p/Sema6d Axis in Viral Myocarditis
}

Runlei Bi

Wannan Medical College

Xueqin Li

Yijishan Hospital of Wannan Medical College

Weiya Pei

Yijishan Hospital of Wannan Medical College

Yingying Zhang

Yijishan Hospital of Wannan Medical College

Kun Lv ( $\nabla$ lvkun315@126.com )

Yijishan Hospital of Wannan Medical College https://orcid.org/0000-0002-1895-6700

Research article

Keywords: IncRNA, miRNA, ceRNA, macrophage polarization, viral myocarditis

Posted Date: July 13th, 2021

DOI: https://doi.org/10.21203/rs.3.rs-684513/v1

License: (9) This work is licensed under a Creative Commons Attribution 4.0 International License. Read Full License 


\section{Abstract}

\section{Background}

Macrophage polarization is vital for coxsackievirus B3 (CVB3)-induced viral myocarditis (VM). However, only a few empirical studies have focused on exploring the relationship between macrophage polarization and VM.

\section{Methods}

Quantitative real-time PCR was used to determine the ex-pression levels of AK141955 and miR-124-3p in $\mathrm{M} 1 / \mathrm{M} 2$ cells. The functional role of macrophage polarization was investigated by in vitro gene silencing assays. Mechanistically, dual-luciferase reporter and RNA immunoprecipitation (RIP) assays were performed to confirm that AK141955 sponged miR-124-3p and alleviated the suppression of target Sema6d expression.

\section{Results}

Functionally, AK141955 knockdown significantly reduced inflammation, inhibited the M1 phenotype, and promoted the M2 phenotype in vitro and in vivo, whereas miR-124-3p inhibitors counteracted these effects. Evidence is presented, showing that AK141955 acted as a "ceRNA" of miR-124-3p to relieve the repressive effect of miR-124-3p on its target Sema6d, then promoted VM development.

Conclusion

Collectively, our findings reveal that AK141955 functions as a pro-inflammatory factor in pro-moting VM progression through the miR-124-3p/Sema6d axis, providing a solid evidence base for treating VM patients.

\section{Background}

Viral myocarditis (VM) is defined as inflammation of the heart muscles; eventually, the disease may progress to dilated cardiomyopathy and heart failure. VM is a leading cause of sudden death in young adults(1). Coxsackievirus B3 (CVB3) has been identified as the most common VM pathogen (2). Recent evidence suggests that overwhelming inflammation is the primary etiologic factor in the course of VM (3). However, the extent to which a lack of inflammation is causally associated with VM remains unclear.

Macrophages differentiate into two distinct phenotypes. M1 macrophages, induced by lipopolysaccharide (LPS) and interferon- $y$ (IFN- $\gamma$ ), typically promote myocardial inflammation and tissue destruction by producing pro-inflammatory cytokines(4). In contrast, M2 macrophages, induced by interleukin 4 (IL-4) or IL-13 secrete anti-inflammatory cytokines associated with tissue repair $(5,6)$. Our research group has shown that M1 macrophages significantly aggravate myocarditis, whereas M2 
macrophages alleviate myocardial inflammation $(7,8)$. Despite the importance of macrophage polarization to inflammation in VM, its regulatory mechanisms are still not fully understood.

With in-depth ncRNA studies, accumulating evidence has revealed the vital roles of ncRNAs in macrophage polarization (9-11). In earlier stages, our research group used gene chip assays to detect changes in IncRNA and miRNA expression and found that IncRNAs AK085865 and miR-155 play an important role in macrophage polarization $(7,12)$. However, the relationship between IncRNAs and miRNAs remains unclear (13-15). We surmise that such regulatory networks may be involved in macrophage polarization (16-18). Therefore, based on the results of bioinformatics and the regulatory rules of ceRNA, we constructed a ceRNA network related to macrophage polarization. However, to date, the significance of ncRNAs in macrophage polarization has been sparsely characterized. Macrophage polarization-associated miRNAs, IncRNAs, and their regulatory networks need to be specifically and thoroughly investigated in cardiovascular diseases.

In this study, we found that AK141955 knockdown diminished M1 phenotypical expression while promoting polarization to the M2 phenotype. Furthermore, mechanistic analysis revealed that AK141955 functions as a ceRNA to regulate the expression and function of Sema6d by competitively binding to miR-124-3p. We also show here that AK141955-knockdown mice had decreased susceptibility to CVB3induced VM. Taken together, AK141955 is a regulator of VM development and progression and is a valuable biomarker and therapeutic target. This is the first report of the relationship between AK141955 and miR-124-3p as miRNA sponges in VM, broadening our understanding of the expression pattern. Therefore, it is of great practical significance to explore the potential pathogenesis of VM and to develop effective treatment strategies.

\section{Methods}

\subsection{Ethics statement}

All animal experimental procedures were approved by the Animal Ethics Committee of Yijishan Hospital (approval number: No. AECYJS-2016002) and were performed according to the Guide for the Care and Use of Laboratory Animals published by the US National Institutes of Health (8th Edition, National Research Council, 2011).

\subsection{Isolation and cultivation of murine BMDMs}

Bone marrow cells were obtained by flushing mice femurs with a modified medium (DMEM)-HEPES medium (Gibco; Thermo Fisher Scientific, Waltham, MA, USA). Cells were collected in 50-mL tubes and centrifuged for $10 \mathrm{~min}(100 \mathrm{~g})$. The supernatant was removed, and the cells were suspended in DMEM ( $20 \%$ FBS; $20 \%$ L929 supernatant). Then, $1 \times 106$ cells were cultured in 6 -well plates at $37^{\circ} \mathrm{C}$ and $5 \% \mathrm{CO} 2$ for $7 \mathrm{~d}$ (BMDMs). Macrophage polarization was obtained by removing the culture medium and culturing cells for an additional $48 \mathrm{~h}$ in DMEM supplemented with $10 \% \mathrm{FBS}$ and $100 \mathrm{ng} / \mathrm{mL}$ LPS (Sigma-Aldrich, St 
Louis, MO, USA) plus 20 ng/mL IFN-y (PeproTech, Inc., Rocky Hill, NJ, USA) (for M1 polarization) or 20 ng/mL IL-4 (PeproTech, Inc.) (for M2 polarization).

\subsection{Knockdown of AK141955 mice were constructed}

AK141955 interference AAV and control AAV, with an AAV9 serotype, were purchased from Shanghai Jikai Gene Chemical Technology Co., Ltd. (Shanghai, China). All AAV vectors were injected through the tail vein at a dose of $1 \times 1011 \mathrm{vg} /$ mouse. AK141955 knockdown (sh-AK141955) and negative control (sh-NC) in mice were performed. The mice were kept under sterile conditions and received sterile feed and water.

\subsection{Virus and myocarditis model}

The original stock of CVB3 (Nancy strain) was a gift from Professor Wei Hou (School of Basic Medical Sciences, Wuhan University) and was maintained by passage through HeLa cells (ATCC number: CCL-2). Virus titer was routinely determined prior to infection by a $50 \%$ tissue culture infectious dose (TCID50) assay of HeLa cell monolayers according to previously published procedures. Mice were infected by an intraperitoneal (ip) injection of $0.1 \mathrm{~mL}$ of phosphate-buffered saline (PBS) containing approximately $1 \times$ 105 plaque-forming units (PFU) of the virus on day 0. Tissue or cells were collected on day 7.

\subsection{Cell culture and reagents}

BMDMs were isolated from the femurs and tibias of adult mice, as previously described. Macrophages were cultured in DMEM complete medium (20\% fetal bovine serum, $20 \%$ L 929 cell supernatant) at $37^{\circ} \mathrm{C}$ and 5\% CO2 for $7 \mathrm{~d}$. M1 and M2 macrophage polarization was obtained by removing the culture medium and culturing cells for an additional $48 \mathrm{~h}$ in DMEM supplemented with $10 \%$ FBS and $100 \mathrm{ng} / \mathrm{mL}$ LPS (Sigma-Aldrich) plus 20 ng/mL IFN-y (PeproTech, Inc.) (for M1 polarization) or 20 ng/mL IL-4 (PeproTech, Inc.) (for M2 polarization).

\subsection{Small interfering RNA and transfection of BMDMs}

The IncRNA Smart Silencer targeting AK141955 at different sites and a negative control (NC) with no definite target were synthesized by RiboBio Co., Ltd., (Guangzhou, China). BMDMs were seeded on 6-well plates at a density of $5 \times 105$ cells/well overnight and then transfected with Smart Silencer or the negative control at a final concentration of $100 \mathrm{nmol} / \mathrm{L}$ using Lipofectamine 3000 (Invitrogen, Waltham, MA, USA). The interfering efficiency was detected by RT-qPCR after transfection, and the Smart Silencer with a silencing efficacy of more than $70 \%$ was selected for further experiments.

\subsection{RT-qPCR}

Total RNA was isolated using an RNeasy Kit (Qiagen, Hilden, Germany), and cDNA was synthesized using an iScript cDNA Synthesis Kit (Bio-Rad Laboratories, Hercules, CA, USA). SYBR Green dye-based quantitative real-time PCR was performed using SYBR Green PCR Master Mix and a CFX96 Real-Time PCR System (Bio-Rad Laboratories). A commercial Hairpin-itTM miRNA qPCR Quantification Kit (RiboBio Co., Ltd.) was used for miRNA real-time PCR. Briefly, 2 ug RNA was used as a template and reversetranscribed using an miRNA-specific RT primer. The resulting cDNA was amplified using a universal 
reverse primer and a specific forward primer. The mRNA or miRNA expression levels were calculated using the comparative CT $(\triangle \triangle \mathrm{CT})$ method and normalized against GAPDH or U6 snRNA levels. All reactions were performed in triplicate. Standard SYBR Green real-time qPCR assays were performed with the following primers: GAPDH, 5'-GGTTGTCTCCTGCGACTTCA-3' and 5'-TGGTCCAGGGTTTCTTACTCC-3'; NOS2, 5'-ATCTTTGCCACCAAGATGGCCTGG-3' and 5'-TTCCTGTGCTGTGCTACAGTTCCG-3'; TNF-a, 5'CCAGTGTGGGAAGCTGTCTT-3' and 5'-AAGCAAAAGAGGAGGCAACA-3'; IL-12, 5'GATGTCACCTGCCCAACTG-3' and 5'-TGGTTTGATGATGTCCCTGA-3'; Arg-1, 5'TGACTGAAGTAGACAAGCTGGGGAT-3' and 5'-CGACATCAAAGCTCAGGTGAATCGG-3'; YM-1, 5'ATGAAGCATTGAATGGTCTGAAAG-3' and 5'-TGAATATCTGACGGTTCTGAGGAG-3'; FIZZ1, 5'AGGTCAAGGAACTTC TTGCCAATCC-3' and 5'- AAGCACACCCAGTAGCAGTCATCCC-3'; AK141955, 5'CGTGTTGTGGTTGTTCTTGTGGTG-3' and 5'-GGATGCTTGATG CCCTGTTCTGG - 3'.

\subsection{RIP}

RIP experiments were performed to verify the relationship between AK141955 and miR-124-3p using an EZ-Magna RIP Kit (MilliporeSigma, Burlington, MA, USA). Briefly, BMDMs were lysed in a complete RIP lysis buffer containing protease and RNase inhibitors. Then, $100 \mu \mathrm{L}$ of the extract was incubated with argonaute2 (AGO2) antibody or control immunoglobulin G (IgG; MilliporeSigma) for $8 \mathrm{~h}$ at $4^{\circ} \mathrm{C}$, followed by the addition of protein $A / G$ beads. Afterward, the beads were incubated with proteinase $K$ to digest the proteins. Finally, the purified RNA was subjected to qRT-PCR analysis using specific primers for AK141955.

\subsection{FACS analysis}

Single-cell suspensions were pooled from heart tissues. Surface markers were stained with fluorochromeconjugated mAbs diluted in 1\% FBS in PBS: iNOS, CD206 and CD11b (eBioscience; BD Pharmingen; Biolegend ). For intracellular staining, cells were fixed and permeabilized using a fixation buffer and permeabilization solution (eBioscience). Cell fluorescence was measured using FACS (Beckman Coulter), and data were analyzed using FlowJo software (BD Biosciences, Franklin Lakes, NJ, USA).

\subsection{Bacteria killing assay}

Briefly, $0.1 \times 106 \mathrm{CFU} / \mathrm{mL}$ Escherichia coli was added to macrophages in 6-well plates and incubated at $37^{\circ} \mathrm{C}$ for $1 \mathrm{~h}$. Supernatants were diluted 100 times, and $100 \mu \mathrm{L}$ of the diluted supernatants were plated on Luria broth agar plates. The plates were incubated overnight at $37^{\circ} \mathrm{C}$, and the bacterial colonies were counted. $\mathrm{CFU} / \mathrm{mL}$ indicates the number of bacterial colonies $\times$ dilution factor/volume of the plated diluted supernatants.

\subsection{Phagocytosis assay}

Briefly, exosomes were labeled with PKH26. Subsequently, $0.5 \times 106$ labeled exosomes suspended in 500 $\mu \mathrm{L}$ media were added to the macrophages, followed by incubation at $37^{\circ} \mathrm{C}$ for $60 \mathrm{~min}$. Macrophages were then washed with cold PBS, resuspended in PBS containing PE-cy7, and analyzed by flow cytometry. 


\subsection{Arginase activity assay}

Myocardial-infiltrating macrophages in sh-AK141955 and sh-NC mice during CVB3-induced VM were prepared. Afterward, $1 \times 106$ macrophages were lysed with $50 \mu \mathrm{L}$ of $0.1 \%$ Triton X-100 for 30 min and then added to $50 \mu \mathrm{L}$ of $50 \mathrm{mmol} / \mathrm{L} \mathrm{Tris-HCl} / 10 \mathrm{mmol} / \mathrm{L} \mathrm{Cl} 2 \mathrm{Mn} \cdot 4 \mathrm{H} 20(\mathrm{pH} \mathrm{7.5})$ and incubated at $55^{\circ} \mathrm{C}$ for 10 min. L-arginine hydrolysis was carried out by incubation with $25 \mu \mathrm{L}$ of $0.5 \mathrm{~mol} / \mathrm{L} \mathrm{L}$-arginine $(\mathrm{pH} 9.7)$ at $37^{\circ} \mathrm{C}$ for $60 \mathrm{~min}$. The reaction was then stopped using $400 \mu \mathrm{L}$ of stop solution [H2SO4 (96\%)/H3PO4 $(85 \%) / \mathrm{H} 20(1: 3: 7, \mathrm{v} / \mathrm{v} / \mathrm{v})]$ and $25 \mu \mathrm{L}$ of $9 \% 2$-isonitrosopropiophenone. The reactions were incubated at $100^{\circ} \mathrm{C}$ for $45 \mathrm{~min}$, and $100 \mu \mathrm{L}$ of each sample was analyzed using a microplate reader at $540 \mathrm{~nm}$.

\subsection{Cytokines ELISA}

Cytokine levels of Arg-1, NOS2, TNF-a, and IL-12 were measured in homogenized heart or cell supernatants using the respective cytokine ELISA kits (R\&D Systems, Inc., McKinley, MN, USA), according to the manufacturer's instructions.

\subsection{Serological Index of Myocarditis}

Serum cTnI measurement was conducted at Yijishan Hospital, Wuhu, China, using a DXI800 immunology analyzer (Beckman Coulter).

\subsection{Hemodynamics analysis}

Seven days after CVB3 injection, the mice were anesthetized with an intraperitoneal injection of $1 \%$ pentobarbital sodium (50 mg/kg). The mice were endotracheally intubated and mechanically ventilated with room air (respiratory rate, 45 breaths/min; respiration ratio, 1:1; tidal volume, $1.5 \mathrm{~mL}$ ). The left ventricular (LV) performance was assessed using a self-made polyethylene catheter inserted via the right carotid artery as previously described. Briefly, the catheter $(0.3 \mathrm{~mm} \times 0.6 \mathrm{~mm} I D / O D)$, which was prefilled with fresh heparinized saline $(500 \mathrm{U} / \mathrm{mL})$, was inserted into the $\mathrm{LV}$ through the cardiac apex. The catheter was connected to a pressure transducer linked to a biological data acquisition system (RM6240, Chengdu, China). After stabilization, the readout signals were stored and displayed on a computer to determine the off-line LV end-diastolic pressure (LVEDP), LV systolic pressure (LVSP), the maximum first derivative of $L V$ pressure (+ dP/dtmax), and minimum first derivative of LV pressure $(-\mathrm{dP} / \mathrm{dttmax})$.

\subsection{Histopathology}

Hearts were cut longitudinally, fixed in $10 \%$ phosphate-buffered formalin, and embedded in paraffin. Sections ( $5 \mu \mathrm{m}$ thick) were cut at various depths in the tissue sections and stained with hematoxylin and eosin (H\&E) to determine the level of inflammation. The sections were examined by two independent investigators who were blinded to the disease state of the mice.

\subsection{Statistical Analysis}


Data are shown as mean \pm SEM. Statistical analysis of the data was performed using the two-tailed independent Student's t-test. Analysis of variance (ANOVA) was conducted using SPSS software (SPSS, Inc., Chicago, IL, USA), and Kaplan-Meier survival curves were drawn using GraphPad Prism v.5.0 (GraphPad Software, San Diego, CA, USA). Statistical significance was considered at $p<0.05$.

\section{Results}

\subsection{Construction of ceRNA network of macrophage polarization.}

Here, we investigated the role of non-coding RNAs in macrophage polarization. Microarray analysis was performed in in vitro-polarized M1 and M2 macrophages to screen for differentially expressed IncRNAs, mRNAs, and miRNAs in macrophage polarization. We observed that the expression of 3,754 IncRNAs, 2,080 mRNAs, and 107 miRNAs was often altered between M1 and M2. TargetScan, PicTar, and miRDB were used to predict the target genes. We will introduce differentially upregulated mRNAs combined with downregulated miRNAs and differentially downregulated mRNAs combined with upregulated miRNAs which were performed for target gene prediction where we obtained miRNA-mRNA analysis results (Additional file 2). Correlation coefficient screening was conducted for the differential mRNA and IncRNA data in mRNA and IncRNA chips to obtain the results of gene co-expression network analysis (Additional file 3). Here, the highly negatively correlated mRNAs and IncRNAs were filtered out, and only highly positively correlated mRNAs and IncRNAs were retained. Then, based on the prediction results of miRNA target genes and the combined analysis results of gene expression, an extensive ceRNA network was constructed (Additional file 1).

Based on the extensive ceRNA network, we reviewed literature on miRNA regulation of macrophage polarization and selected miRNAs with the most nodes to construct a new ceRNA network. The blue round nodes represent miRNAs, the green square nodes represent mRNAs, and the orange nodes represent IncRNAs. T-shaped edges indicate miRNA inhibition of target genes, and pure linear edges indicate ceRNA relationships (Fig. 1A). Based on the miRNA microarray analysis results, we verified the differentially expressed miRNAs by $\mathrm{qPCR}$, and results showed that the qPCR verification results of miR33-3p, miR-124-3p, and miR-141-3p were consistent with the microarray results, with relatively high expressions in M1. The results of qPCR validation for miR-3094-3p were not statistically significant (Fig. 1B). Based on the results shown in Fig. 2, we selected IncRNAs related to miR-33-3p, miR-124-3p, and miR-141-3p and verified the microarray analysis results using qPCR. We found that most IncRNAs were highly expressed in M2, which was consistent with the microarray results (Fig. 1C).

\subsection{Preliminary analysis of miRNA function in vitro}

In order to screen core miRNAs, we observed the changes in M1 surface marker NOS2 and M2 surface marker Arg-1 through overexpression and inhibition of miRNAs to clarify the role of miRNAs in macrophage polarization. After miR-33-3p and miR-124-3p overexpression, the expression of surface 
marker NOS2 of M1 was downregulated, while after miR-141-3p overexpression, the expression of surface marker NOS2 of M1 was not statistically significant (Fig. 2A). After miR-33-3p and miR-141-3p overexpression, the expression of M2 surface marker Arg-1 was downregulated, while after miR-124-3p overexpression, the expression of M2 surface marker Arg-1 was increased (Fig. 2B). After miR-33-3p and miR-141-3p inhibition, the expression of surface marker NOS2 of M1 was downregulated, while after miR124-3p inhibition, the expression of surface marker NOS2 of M1 was increased (Fig. 2C). After miR33-3p and miR-124-3p inhibition, the expression of M2 surface marker Arg-1 was downregulated, while after miR-141-3p inhibition, the expression of M2 surface marker Arg-1 was upregulated (Fig. 2D). We found that upregulation of miR-124-3p could inhibit the M1 phenotype and promote the M2 phenotype, while downregulation of miR-124-3p could promote the M1 phenotype and inhibit the M2 phenotype. In conclusion, miR-124-3p promoted macrophage polarization to M2.

\subsection{AK141955 was screened out as a key IncRNA for miR- 124-3p}

To select key IncRNAs for further research. The nuclear and cytosolic RNAs were isolated from BMDMs, and qPCR data showed that AK141955 was highly expressed in the cytosol than in the nucleus (Fig. 3A). As a control, GAPDH mRNA was specifically located in the cytosol, whereas U6 RNA was primarily located in the nucleus (Fig. 3A). In order to clarify the correlation between miR-124-3p and IncRNA expression, we overexpressed miR-124-3p and found that AK141955 expression was downregulated most noticeably compared with the control group, which was downregulated by 0.2 -fold that of the original (Fig. 3B). When inhibiting miR-124-3p, AK141955 was upregulated by 2.4-fold compared with the control group (Fig. 3C). In conclusion, these results suggest that there may be a negative correlation between miR-124$3 p$ and AK141955.

\subsection{IncRNA AK141955 functions as a ceRNA and sponges miR-124-3p in BMDMs.}

Many researchers have reported that IncRNAs have assumed the role of an miRNA sponge in macrophage polarization. We performed a series of experiments to further explore the interaction among AK141955, miR-124-3p, and Sema6d. A dual-luciferase reporter assay was used to confirm the bioinformatics prediction analysis. The results indicated that the luciferase activity of the Sema6d-WT group was significantly decreased in the miR-124-3p mimic group, and there was no difference between the miR-124-3p mimics and NC groups in the luciferase activity of the Sema6d-MUT group. These results suggest that a direct interaction might exist between Sema6d and miR-124-3p (Fig. 4A). The results indicated that the luciferase activity of the AK141955-WT group was significantly decreased in the miR124-3p mimic group, and there was no difference between the miR-124-3p mimics and NC groups in the luciferase activity of the AK141955-MUT group. These results suggest that a direct interaction might exist between AK141955 and miR-124-3p (Fig. 4B). It is widely acknowledged that miRNAs regulate target gene expression by binding to Argonaute 2 (Ago2), an essential component of the RNA-induced silencing complex (RISC). Subsequently, RNA immunoprecipitation (RIP) assays were performed in BMDMs to pull 
down the RNA transcripts bound to Ago2. qPCR assays indicated that AK141955 and Sema6d were efficiently pulled down by anti-Ago2 compared with those in the input control. Moreover, AK141955 and Sema6d were highly enriched in cells transfected with miR-124-3p mimics than those in the NC group (Figs. 4C and 4D). Next, qPCR assays demonstrated that AK141955 silencing significantly decreased the mRNA level of Sema6d, whereas inhibition of miR-124-3p increased the mRNA level of Sema6d. In addition, the co-transfection of si-AK141955 and the miR-124-3p inhibitor might eliminate the effects in BMDMs (Fig. 4E). We used qPCR to detect changes in macrophage phenotypes and found that after cotransformation of the miR-124-3p inhibitor and AK141955 silencing, the changes in the expression of M1 marker NOS2 and M2 marker Arg-1 were more distinct. Compared with miR-124-3p alone or IncRNA AK 141955 knockdown alone, the expression level was restored (Fig. 4F). Collectively, these data demonstrate that AK141955 acts as a sponge for miR-124-3p, thus promoting Sema6d expression in BMDMs.

\subsection{AK141955, miR-124-3p and Sema6d functions in macrophage polarization}

To clarify the function of AK141955, miR-124-3p, and Sema6d in macrophage polarization, AK141955 expression in BMDMs was knocked down by AK141955 Smart Silencer. AK141955 silencing in BMDMs diminished M1 phenotypic expression induced by LPS and IFN-y (Fig. 5A) but promoted M2 marker expression induced by IL-4 (Fig. 5B). We used miR-124-3p inhibitors to block miR-124-3p expression in BMDMs. Inhibition of miR-124-3p in BMDMs promoted M1 phenotypic expression induced by LPS and IFN-Y (Fig. 5C) but promoted M2 marker expression induced by IL-4 (Fig. 5D). Sema6d expression in BMDMs was knocked down using a Sema6d Smart Silencer. Sema6d silencing in BMDMs diminished M1 phenotypical expression induced by LPS and IFN-y (Fig. 5E) but promoted M2 marker expression induced by IL-4 (Fig. 5F). These data suggest that AK141955 and Sema6d play a promoting role in M1 macrophage polarization and an inhibiting role in M2 polarization. In contrast, miR-124-3p inhibited the polarization of M1 macrophages and promoted the polarization of M2 macrophages. In summary, cell function experiment results verified that miR-124-3p functions opposed those of IncRNA and Sema6d.

\subsection{AK141955, miR-124-3p, and Sema6d regulate cellular functions associated with M1 and M2 phenotypes.}

M1 macrophages possess high bactericidal activity. To determine whether AK141955, miR-124-3p, and Sema6d regulate bactericidal activity, we knocked down AK141955 or Sema6d using Smart Silencer. The results showed that silencing AK141955 or Sema6d significantly diminished the bactericidal activity of BMDMs, consistent with the suppressive effects of silencing AK141955 or Sema6d on M1 macrophage polarization (Fig. 6 and C). BMDMs transfected with NC or an miR-124-3p mimic (Fig. 6B) showed that overexpression of miR-124-3p significantly diminished the bactericidal activity of BMDMs, consistent with the suppressive effects of miR-124-3p on M1 macrophage polarization. M2 macrophages are associated with a greater ability to engulf apoptotic cells than M1 macrophages. We found that silencing 
AK141955 or Sema6d significantly enhanced the ability of BMDMs to ingest apoptotic cells (Fig. 6 and F). In contrast, miR-124-3p overexpression significantly enhanced the ability of BMDMs to ingest apoptotic cells (Fig. 6E). These findings are congruent with the general effects of AK141955, miR-124-3p, and Sema6d on the expression of M1 and M2 phenotypes.

\subsection{IncRNA AK141955 are more susceptible to CVB3- induced VM.}

As macrophages are the key VM regulators, we attempted to determine the role of AK141955 in VM development. It has been established that shRNA can efficiently knock down the RNA levels of target genes. Sh-NC or sh-AK141955 were intravenously injected into the mice via the lateral tail vein. Seven days later, sh-NC or sh-AK141955 mice were infected with CVB3. On day 7 after infection, AK141955 knockdown alleviated myocardial inflammation compared with the control group, reflected by restricted inflammation foci and a decreased inflamed area (Fig. 7A). AK141955 knockdown significantly improved the survival rate from 30 to $60 \%$ after CVB3 infection (Fig. 7B). Consistent with this observation, AK141955 knockdown significantly attenuated heart weight and attenuated cTnl levels in the serum (Figs. 7C and D). In addition to increasing the survival rate, AK141955 knockdown improved cardiac function in mice that survived CVB3 infection (Fig. 7E). Consistently, F4/80 + macrophages derived from AK141955-knockdown mice also showed high arginase activity and decreased expression of the M1specific genes NOS2, TNF-a, and IL-12, compared with macrophages isolated from the control group mice (Figs. 7F and G). We further examined whether AK141955 knockdown affected the phenotype of heartinfiltrating macrophages. Flow cytometry revealed a significantly lower number of M1 macrophages in AK141955-knockdown mice, as manifested by NOS2 expression (Fig. 7H). These findings suggest that AK141955 knockdown in macrophages was the main reason for the decreased VM susceptibility induced by CVB3.

\section{Discussion}

IncRNAs are non-coding RNAs that do not encode proteins but possess important biological activity (19, 20). Accumulating evidence suggests that IncRNAs are crucial non-coding RNAs involved in regulating cancer, autoimmunity, and inflammation(21). Over the past few years, IncRNAs have been demonstrated to be involved in VM with abnormal expression or dysregulated functions, particularly in inflammatory cells such as macrophages $(22,23)$.

In the present study, we first analyzed the expression profiles of IncRNAs, miRNAs, and mRNAs in M1 and M2 macrophages (BMDMs) using microarray assays. By validating microarray data using qPCR followed by bioinformatics, we constructed a ceRNA network with IncRNAs, miRNAs, and mRNAs. Second, from a functional study in vitro, we chose miR-124-3p as a candidate miRNA. Third, overexpression and inhibition of miR-124-3p showed the most evident changes in AK141955 expression. More importantly, nucleoplasmic separation experiments showed that IncRNA AK141955 was mainly located in the 
cytoplasm. Thus, we identified AK141955-miR-124-3p-Sema6d as a key regulator of macrophage polarization.

To date, IncRNAs have been reported to play an active role in the cytoplasm, where they act as miRNA sponges, interact with RBP, and are involved in protein translation $(24,25)$. Given that most IncRNAs contain miRNA response elements (MREs), accumulating research has shown that the ceRNA mechanism represents the primary approach through which IncRNAs perform their biological functions $(26,27)$. For example, IncRNA IGHCY1 acts as a ceRNA to regulate macrophage inflammation via the miR6891-3p/TLR4 axis in osteoarthritis. Moreover, APF IncRNA regulates autophagy and myocardial infarction by targeting miR-188-3p (28). Sequence information on IncRNA AK141955, miR-124-3p, and Sema6d was obtained from miRDB, NCBI, or other databases, and it was confirmed that IncRNA AK141955 and Sema6d contained binding sites of the miR-124-3p seed region via SeedV1.1 prediction. Thus, experiments including dual-luciferase reporter and RIP assays confirmed that AK141955 colocalized with miR-124-3p in the cytoplasm of macrophages, while AK141955 and miR-124-3p directly bound to miR-124-3p and Sema6d, respectively. IncRNA AK141955 silencing inhibited the expression of Sema6d while miR-124-3p promoted Sema6d expression. At the same time, when silencing AK141955 and inhibiting miR-124-3p, the expression of Sema6d tended to be between them. Rescue experiments also confirmed that AK141955 reversed the effect of miR-124-3p on M2 polarization. Given the above evidence, our results suggest the importance of IncRNA AK141955 as a novel biomarker and therapeutic target in macrophage polarization.

We found that silencing AK141955 and Sema6d suppressed the expression of the M1 phenotype but promoted basal expression of the M2 marker. In contrast, inhibition of miR-124-3p promoted the expression of the M1 marker but suppressed the expression of the M2 marker. We showed that AK141955, miR-124-3p, and Sema6d not only regulate the expression of M1 and M2 phenotypic markers but also control M1/M2-associated macrophage functions. Increasing miR-124-3p diminished the bactericidal activity of macrophages, a typical property associated with M1 macrophages, whereas it enhanced the phagocytic activity for apoptotic cell ingestion, an ability observed to be greater in M2 macrophages. In contrast, AK141955 and Sema6d silencing decreased the bactericidal activity of macrophages and enhanced their ability to phagocytose apoptotic cells $(29,30)$. Our data suggest that the bactericidal and phagocytic activities of AK141955, miR-124-3p, and Sema6d were consistent with the polarization direction of macrophages.

During CVB3-induced VM, macrophages are among the predominant cell types and display functional heterogeneity with pro-inflammatory M1 macrophages or anti-inflammatory M2 macrophages (8). Research by our group and others suggests that the excessive presence of M1 macrophages may cause damage to the host after CVB3 infection, whereas M2 macrophages protect against CVB3-induced VM (7). M1 macrophages express TNF-a, NOS2, and IL-12, induce a strong pro-inflammatory reaction, and contribute to VM. In contrast, M2 macrophages express IL-10 and Arg1 instead of NOS2, depleting arginine stores so that $\mathrm{NO}$ is not produced, rather producing polyamine and proline, which are important for cell differentiation and inflammatory responses (31). 
Using loss of function approaches, we obtained an important understanding of AK141955 biological function at both the cellular and tissue levels. As expected, AK141955 knockdown in BMDMs diminished M1 phenotypic expression but promoted M2 marker expression-AK141955 knockdown inhibits inflammation in vivo. AK141955-knockdown mice had reduced mortality during CVB3-induced VM. Our study also showed reduced cTnl levels in the myocardium of AK141955-knockdown mice compared with control mice, which might be the cause of M2 polarization. We found differences in the phenotypes of heart-infiltrating macrophages when we compared AK141955 knockdown and control mice after CVB3 infection. Compared with the control mice, M1 decreased and M2 increased in AK141955-knockdown mice. Our work showed that macrophages in AK141955-knockdown mice differentiated into the M2 phenotype after CVB3 infection, which could effectively suppress VM development. Combined with the results of studies at the cellular level, IncRNA AK141955 may act as a ceRNA for miR-124-3p to regulate Sema6d expression and promote VM development. Although we are not sure that all these effects are solely due to the absence of AK141955 expression in the heart, our data provide convincing genetic evidence for the in vivo functions of AK085865 in VM development.

To the best of our knowledge, this is the first study to thoroughly investigate the expression, regulation, and function of IncRNA AK141955 in VM. Moreover, this is the first study to investigate the relationship between miR-124-3p and Sema6d. These findings may shed light on VM treatment. However, there are several limitations to the interpretation of our study results. First, our study demonstrated the ability of AK141955 to bind to miR-124-3p; however, there might be other miRNAs that bind to AK141955 to regulate the progression and development of macrophage polarization, which were not predicted by bioinformatics analyses. Second, whether AK141955 regulates VM development through other mechanisms, such as interacting with RNA-binding proteins and sponging trans-acting elements, requires further investigation. Third, the use of a simple M1/M2 model to describe macrophage polarization in vivo is debatable. Therefore, a deeper understanding of the therapeutic potential of AK141955 in VM requires further exploration.

\section{Conclusions}

In conclusion, our findings provide comprehensive preliminary evidence that AK141955 and Sema6d promote the polarization of M1 macrophages, and miR-124-3p promotes the polarization of M2 macrophages. Furthermore, we demonstrated that AK141955 exerted a bona fide regulatory role in blocking the activity of miR-124-3p, thereby controlling inflammatory responses by modulating macrophage polarization. Therefore, AK141955, as an important node of the molecular circuit, can prevent the spontaneous activation of macrophages and potentially affects inflammation and immune diseases. Our results not only explain the potential mechanisms related to IncRNA in the regulation of macrophage polarization progression but also provide a novel potential therapeutic target for patients with VM.

\section{Abbreviations}


IncRNA: long non-coding RNA; ceRNA: competing endogenous RNA; RIP: RNA immunoprecipitation; VM: Viral myocarditis; CVB3: Coxsackievirus B3; LPS: lipopolysaccharide; IFN- $\gamma$ : interferon- $\gamma$; IL-4: interleukin 4; IL-13: interleukin 4; RISC: RNA-induced silencing complex; BMDMs: Bone marrow-derived macrophage; MREs: miRNA response elements; DMEM: modified medium; AGO2: argonaute2; IgG: immunoglobulin G; LVEDP: left ventricular end-diastolic pressure; LVSP: left ventricular systolic pressure;

\section{Declarations}

Acknowledgments: We thank Elsevier for its linguistic assistance during the preparation of this manuscript.

Funding: This work was supported by the National Natural Science Foundation of China (81472017, 81772180, 81870017 and 81701557) and Key Projects of Natural Science Research of Universities in Anhui Province (KJ2019A0413 and KJ2018A0265), and funding from "Peak" Training Program for Scientific Research of Yijishan Hospital, Wannan Medical College (GF2019T01 and GF2019G09).

Availability of data and materials: The Gene Expression Omnibus (GEO) database accession number for the IncRNA profile data of M1 and M2 macrophages reported in this paper is GSE12 5510. The Gene Expression Omnibus (GEO) database accession number for the gene profile data of M1 and M2 macrophages reported in this paper is GSE81992

Competing interests: The authors declare no conflict of interest.

Authors' contributions: K. L. and Y. Z conceived and supervised the project. R.B. wrote the manuscript. R.B., X.L. and W.P. performed the experiments and analyzed the data. All authors approved the final version of the manuscrip

Author details: 1Key Laboratory of Non-coding RNA Transformation Research of Anhui Higher Education Institutes, Wuhu, China. 2Graduate school, Wannan Medical College, Wuhu, China. 3Central Laboratory, The First Affiliated Hospital of Wannan Medical College, Wuhu, China.

4Department of Laboratory Medicine, The First Affiliated Hospital of Wannan Medical College, Yijishan Hospital

\section{References}

1. Schwaab B, et al. (2021) Viral myocarditis: a forbidden indication for cardiac rehabilitation? Eur J Prev Cardiol.

2. Zhang $Y$, et al. (2021) MicroRNA-30a-5p silencing polarizes macrophages toward M2 phenotype to alleviate cardiac injury following viral myocarditis by targeting SOCS1. Am J Physiol Heart Circ Physiol 320: H1348-H1360. 
3. Li Z, et al. (2021) The expression of STAT3 inhibited the NF-KappaB signalling pathway and reduced inflammatory responses in mice with viral myocarditis. Int Immunopharmacol 95: 107534.

4. Liu Z, et al. (2020) Toll-like receptor 4 plays a key role in advanced glycation end products-induced M1 macrophage polarization. Biochem Biophys Res Commun 531: 602-608.

5. Zhou D, Ji L, Chen Y. (2020) TSPO Modulates IL-4-Induced Microglia/Macrophage M2 Polarization via PPAR-gamma Pathway. J Mol Neurosci 70: 542-549.

6. Ye W, Wang J, Lin D, Ding Z. (2020) The immunomodulatory role of irisin on osteogenesis via AMPK-mediated macrophage polarization. Int J Biol Macromol 146: 25-35.

7. Zhang Y, et al. (2016) Silencing MicroRNA-155 Attenuates Cardiac Injury and Dysfunction in Viral Myocarditis via Promotion of M2 Phenotype Polarization of Macrophages. Sci Rep 6: 22613.

8. Zhang Y, et al. (2020) Long non-coding RNA AK085865 ablation confers susceptibility to viral myocarditis by regulating macrophage polarization. J Cell Mol Med 24: 5542-5554.

9. Zhu X, et al. (2021) LncRNA H19 regulates macrophage polarization and promotes Freund's complete adjuvant-induced arthritis by upregulating KDM6A. Int Immunopharmacol 93: 107402.

10. Wang Z, et al. (2021) LncRNA MIAT downregulates IL-1beta, TNF-a to suppress macrophage inflammation but is suppressed by ATP-induced NLRP3 inflammasome activation. Cell Cycle 20: 194203.

11. Wang W, Guo ZH. (2020) Downregulation of IncRNA NEAT1 Ameliorates LPS-Induced Inflammatory Responses by Promoting Macrophage M2 Polarization via miR-125a-5p/TRAF6/TAK1 Axis. Inflammation 43: 1548-1560.

12. Zhang Y, et al. (2020) IncRNA AK085865 Promotes Macrophage M2 Polarization in CVB3-Induced VM by Regulating ILF2-ILF3 Complex-Mediated miRNA-192 Biogenesis. Mol Ther Nucleic Acids 21: 441451.

13. Zhou XB, et al. (2020) LncRNAGAS5 sponges miRNA-221 to promote neurons apoptosis by upregulated PUMA under hypoxia condition. Neurol Res 42: 8-16.

14. Yang Y, et al. (2021) Targeted silver nanoparticles for rheumatoid arthritis therapy via macrophage apoptosis and Re-polarization. Biomaterials 264: 120390.

15. Xian S, Ding R, Li M, Chen F. (2021) LncRNA NEAT1/miR-128-3p/AQP4 axis regulating spinal cord injury-induced neuropathic pain progression. J Neuroimmunol 351: 577457.

16. Zhang P, et al. (2020) LncRNA NEAT1 Sponges MiRNA-148a-3p to Suppress Choroidal Neovascularization and M2 macrophage polarization. Mol Immunol 127: 212-222. 
17. Wang Z, Fu M, Li Y. (2020) miR-142-5p and miR-212-5p cooperatively inhibit the proliferation and collagen formation of cardiac fibroblasts by regulating c-Myc/TP53INP1. Can J Physiol Pharmacol 98: 314-323.

18. Nie K, et al. (2020) A novel ceRNA axis involves in regulating immune infiltrates and macrophage polarization in gastric cancer. Int Immunopharmacol 87: 106845.

19. Zhong L, Zhen M, Sun J, Zhao Q. (2021) Recent advances on the machine learning methods in predicting ncRNA-protein interactions. Mol Genet Genomics 296: 243-258.

20. Wang J, et al. (2021) EDLMFC: an ensemble deep learning framework with multi-scale features combination for ncRNA-protein interaction prediction. BMC Bioinformatics 22: 133.

21. Zhang J, et al. (2021) LncRNA HLA-F-AS1 promotes colorectal cancer metastasis by inducing PFN1 in colorectal cancer-derived extracellular vesicles and mediating macrophage polarization. Cancer Gene Ther.

22. Zhang N, Sun Y. (2019) LncRNA ROR facilitates myocardial fibrosis in rats with viral myocarditis through regulating C-Myc expression. Eur Rev Med Pharmacol Sci 23: 10982-10988.

23. Xue YL, et al. (2020) Long non-coding RNA MEG3 inhibits M2 macrophage polarization by activating TRAF6 via microRNA-223 down-regulation in viral myocarditis. J Cell Mol Med 24: 1234112354.

24. Zhang P, et al. (2020) IncRNA IGHCgamma1 Acts as a ceRNA to Regulate Macrophage Inflammation via the miR-6891-3p/TLR4 Axis in Osteoarthritis. Mediators Inflamm 2020: 9743037.

25. Liu Z, et al. (2020) LncRNA MALAT1 prevents the protective effects of miR-125b-5p against acute myocardial infarction through positive regulation of NLRC5. Exp Ther Med 19: 990-998.

26. Chen J, et al. (2020) LncRNA TP73-AS1/miR-539/MMP-8 axis modulates M2 macrophage polarization in hepatocellular carcinoma via TGF-beta1 signaling. Cell Signal 75: 109738.

27. Chen H, Xia W, Hou M. (2020) Correction to: LncRNA-NEAT1 from the competing endogenous RNA network promotes cardioprotective efficacy of mesenchymal stem cell-derived exosomes induced by macrophage migration inhibitory factor via the miR-142-3p/FOX01 signaling pathway. Stem Cell Res Ther 11: 376.

28. Wang $\mathrm{K}$, et al. (2015) APF IncRNA regulates autophagy and myocardial infarction by targeting miR-188-3p. Nat Commun 6: 6779.

29. Banerjee S, et al. (2013) MicroRNA let-7c regulates macrophage polarization. J Immunol 190: 6542-6549. 
30. Banerjee $\mathrm{S}$, et al. (2013) miR-125a-5p regulates differential activation of macrophages and inflammation. J Biol Chem 288: 35428-35436.

31. Rong J, et al. (2020) Inhibition of let-7b-5p contributes to an anti-tumorigenic macrophage phenotype through the SOCS1/STAT pathway in prostate cancer. Cancer Cell Int 20: 470.

\section{Figures}

A

D130075C08 B130042H10
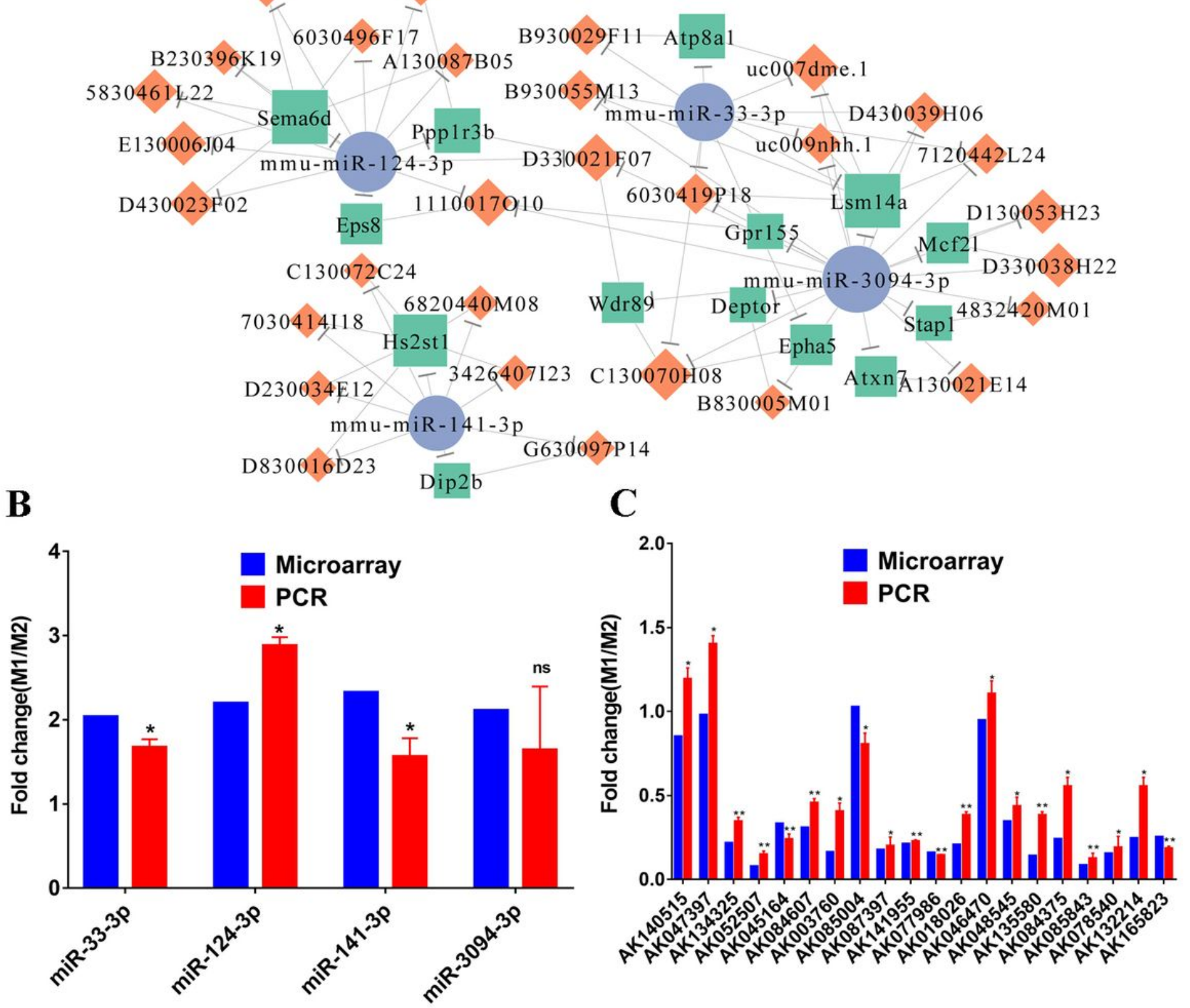

Figure 1

Quantitative real-time PCR validation for the microarray data. (A) LncRNA-miRNA-mRNA networks. The circle represents miRNA, the green square figure represents IncRNA, the orange square figure represents mRNA. (B) Validation of miRNA microarray by qPCR. (C) Validation of IncRNA microarray by qPCR. Data 
are reported as mean \pm s.d. of replicate analyses. * $p<0.05, * \star p<0.01$, ns indicates no significance based on Student's t-test.
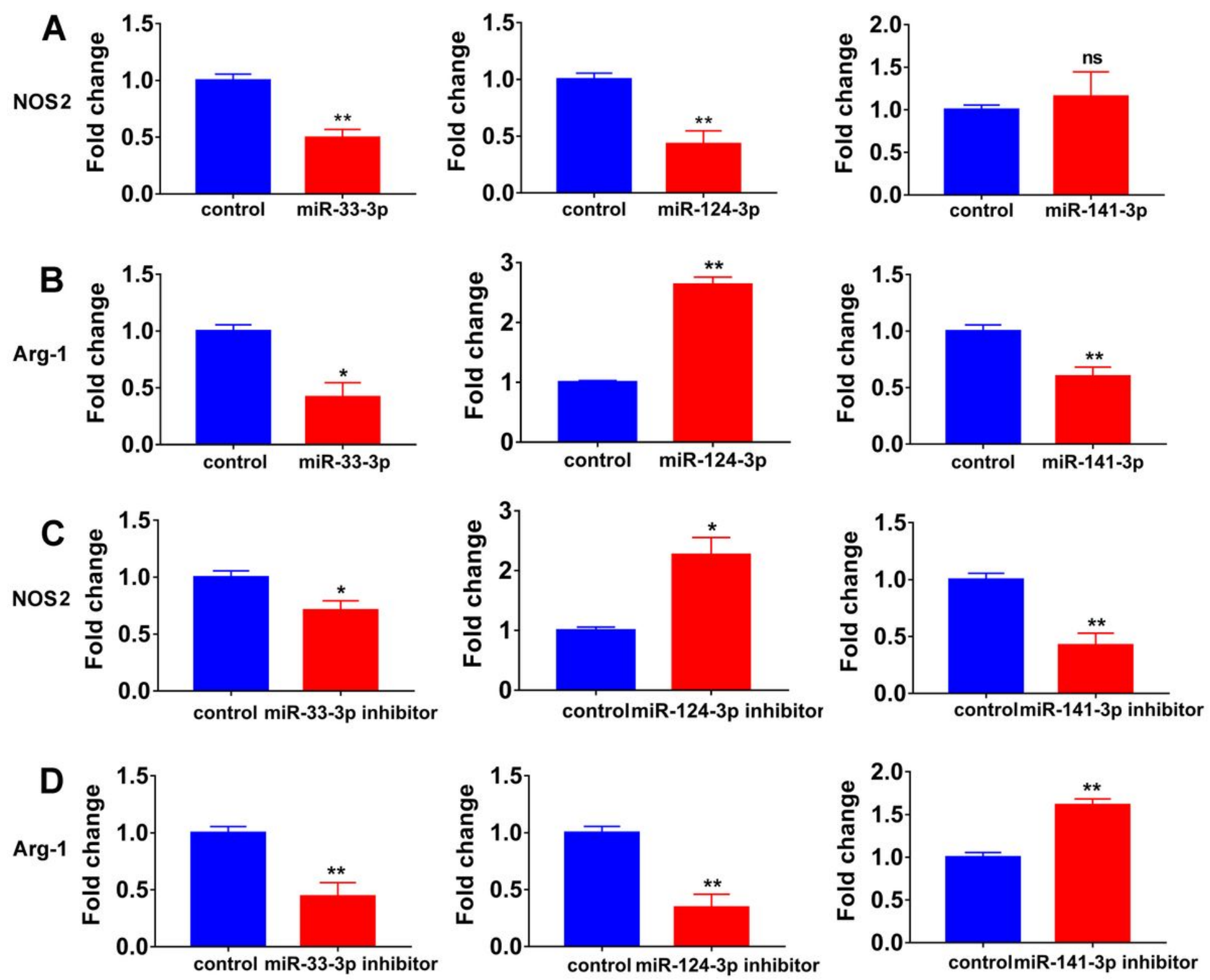

Figure 2

The effect of miRNA on macrophage polarization in vitro. (A) NOS2 levels were determined by overexpressing miRNA. (B) Arg-1 levels were determined by overexpressing miRNA. (C) NOS2 levels were determined by inhibiting miRNA. (D) Arg-1 levels were determined by inhibiting miRNA. Data are reported as mean \pm s.d. of replicate analyses. ${ }^{*} p<0.05, * \star p<0.01$, ns indicates no significance based on Student's t-test. 


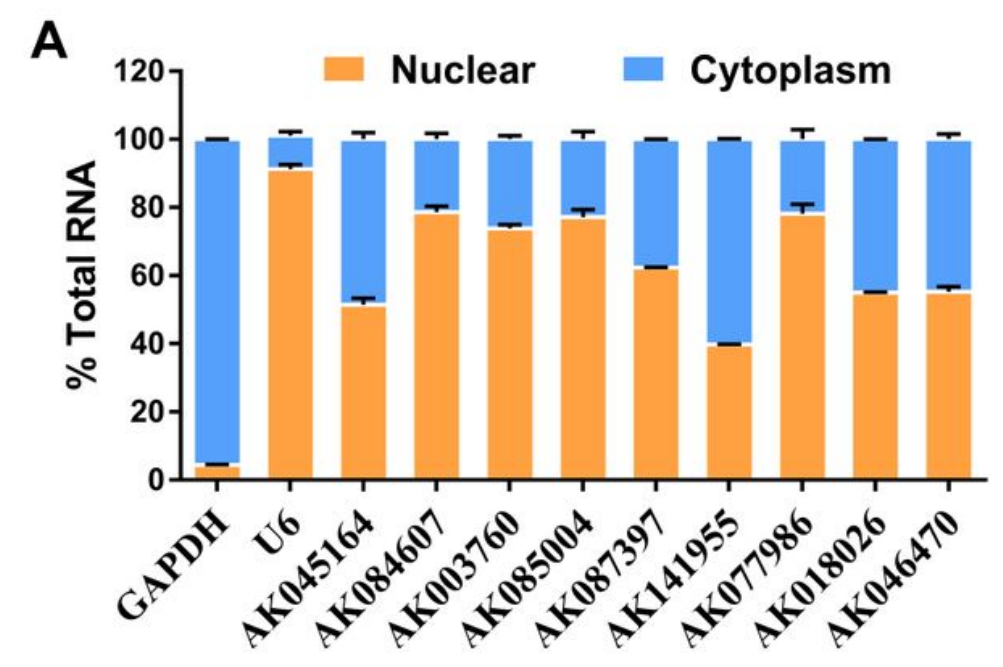

B
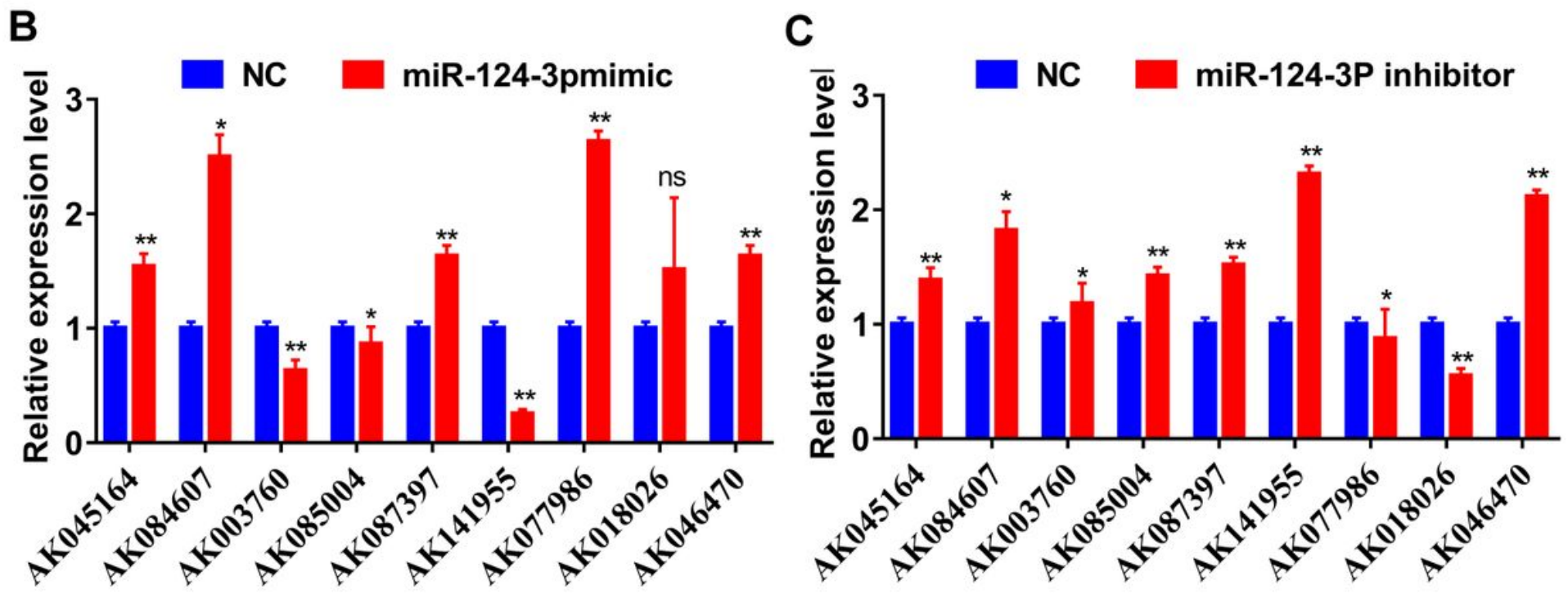

Figure 3

Analysis of characteristics of miR-124-3p-related IncRNA in BMDMs. (A) qPCR analysis of RNA extracted from the nucleus (yellow) and cytosol (blue) in BMDMs. (B) Changes in IncRNA were observed with overexpressed miR-124-3p. (C) Changes in IncRNA were observed by inhibiting miR-124-3p. Data are reported as mean \pm s.d. of replicate analyses. ${ }^{*} p<0.05$, ${ }^{\star *} p<0.01$, ns indicates no significance based on Student's t-test. 
A

Sems6d-WT 5'...-TTTTGTAACATTGTGCTGCCTTAG -...3' miR-124-3P 3'-CCGUAAGUGGCGCACGGAAU-5'

Sema6d-MUT 5'...-UUUUGUAACAUUGUGCACGGAAUG-....

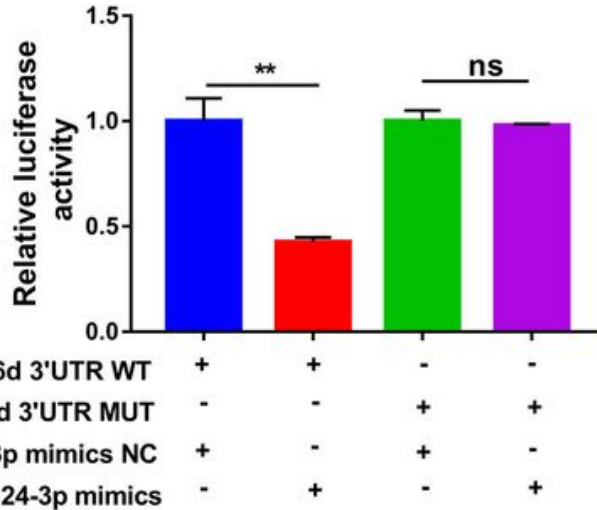

C

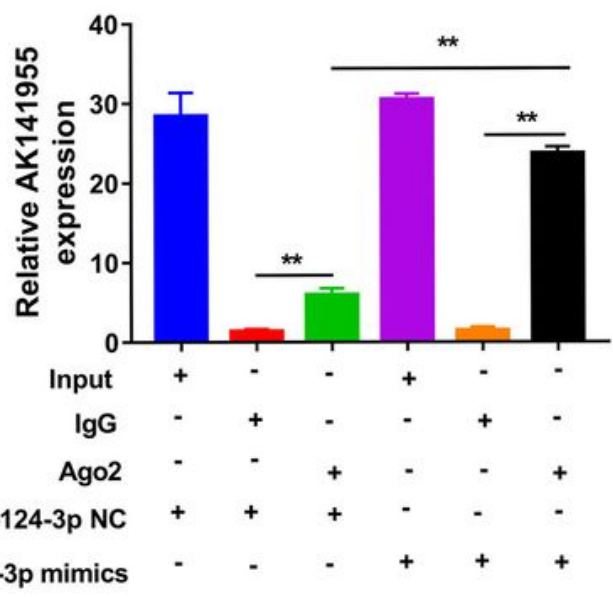

$\mathbf{E}$

NC
si-AK141955 miR-124-3p inhibitor
si-AK141955 +miR-124-3p inhibitor

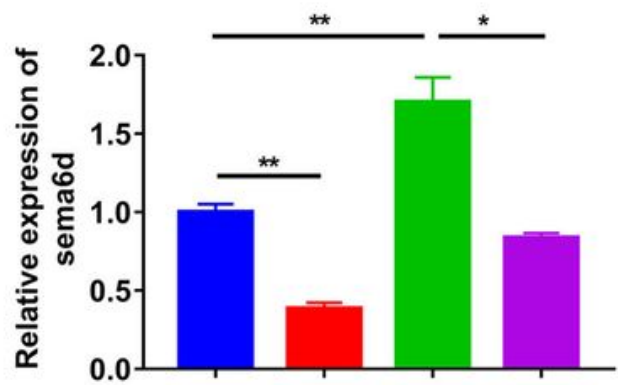

B

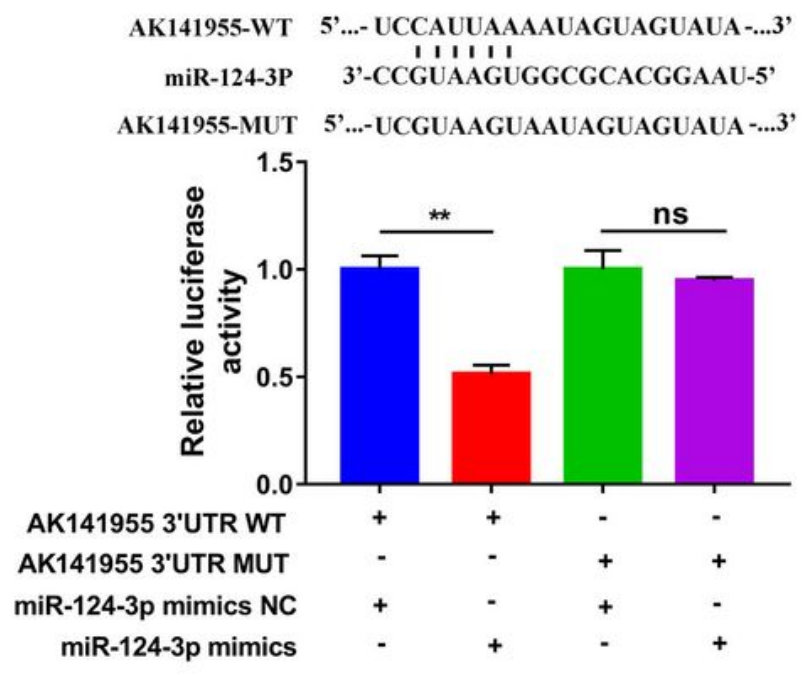

D

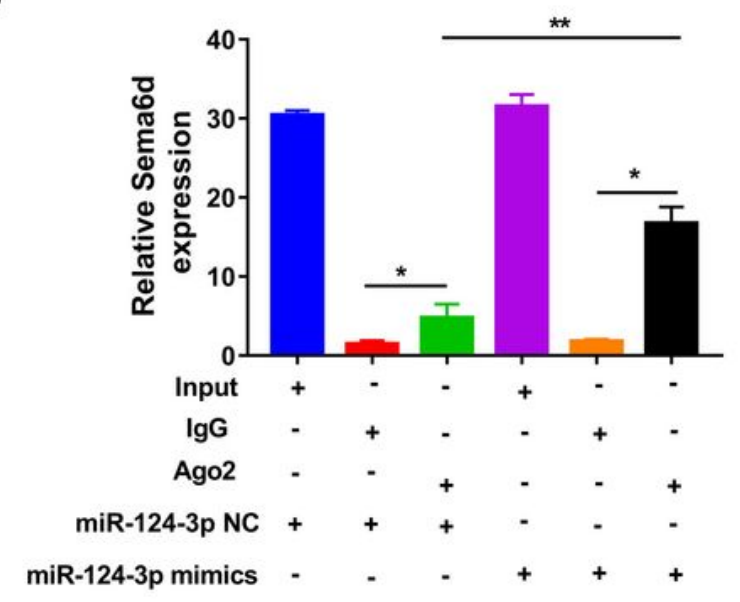

$\mathbf{F}$
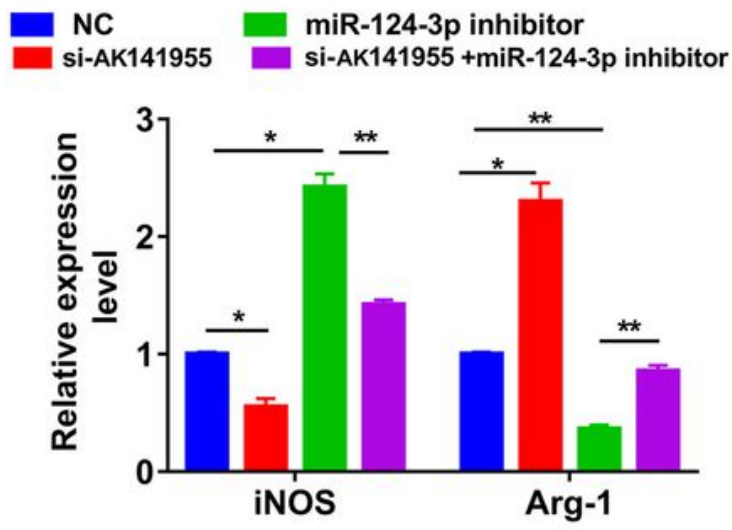

Figure 4

AK141955 serves as an miRNA sponge for miR-124-3p to regulate sema6d expression. (A) Relative luciferase activities were detected in 293T cells after co-transfection with sema6d-WT or sema6d-MUT, and miR-124-3p mimics or mimics NC. (B) Relative luciferase activities were detected in 293T cells after co-transfection with AK141955-WT or AK141955-MUT, and miR-124-3p mimics or mimics NC. (C) An antiAgo2 RIP assay was executed in BMDM after transfection with mimics and NC, followed by qPCR to 
detect AK141955. (D) The relative expression of sema6d was detected in BMDM transfected with NC, silenced AK141955, and miR-124-3p inhibitor using RT-qPCR. (D) An anti-Ago2 RIP assay was executed in BMDM after transfection with mimics and NC, followed by qPCR to detect Sema6d. (E) The relative expression of sema6d was detected in BMDM transfected with NC, silenced AK141955, and miR-124-3p inhibitor using qPCR. (F) The relative expression level of NOS2 and Arg-1 were detected in BMDM transfected with NC, silenced AK141955, and miR-124-3p inhibitor using qPCR. Data are reported as mean \pm s.d. of replicate analyses. ${ }^{*} p<0.05,{ }^{*} p<0.01$, ns indicates no significance based on Student's ttest. 

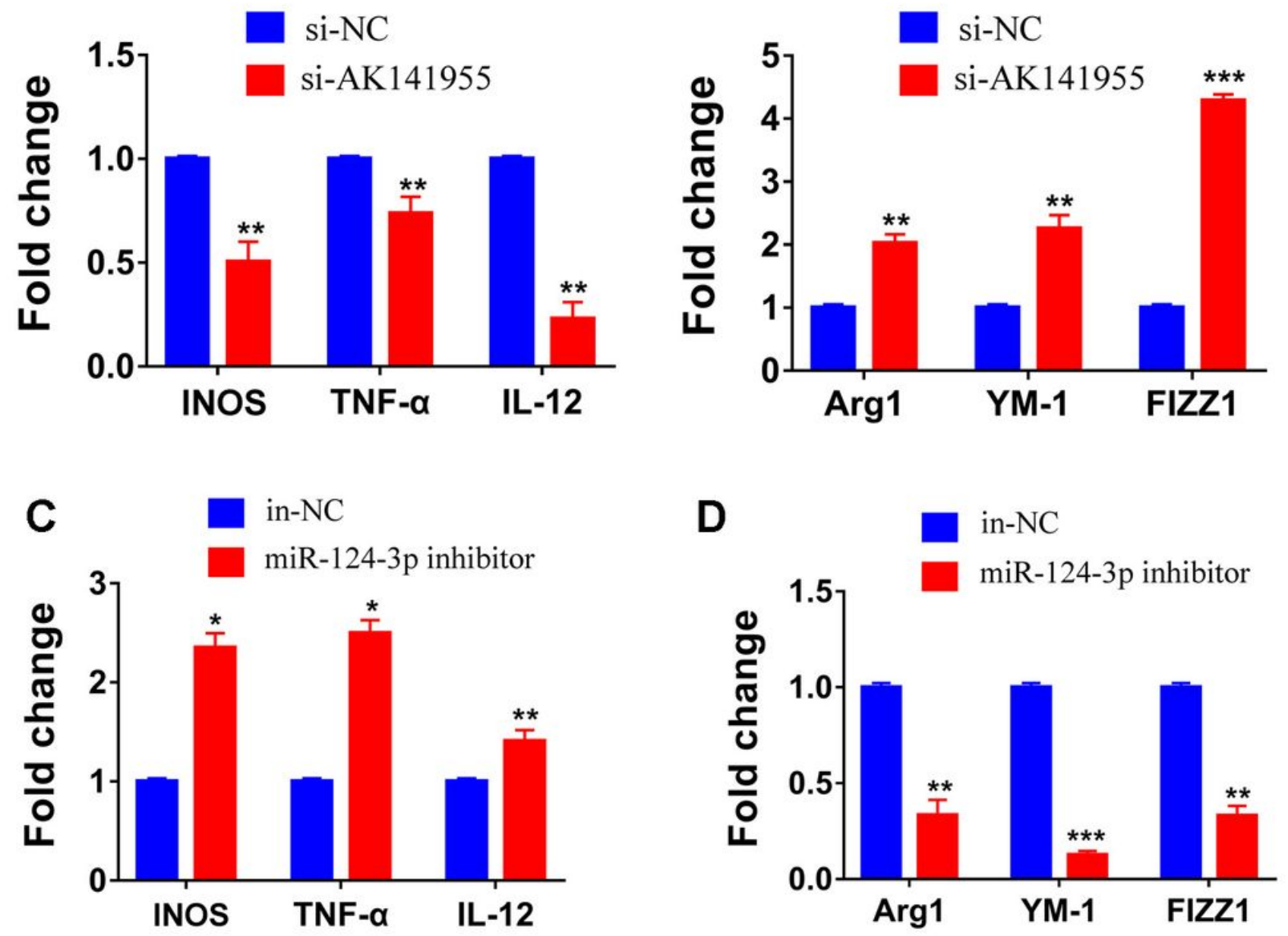

E

F
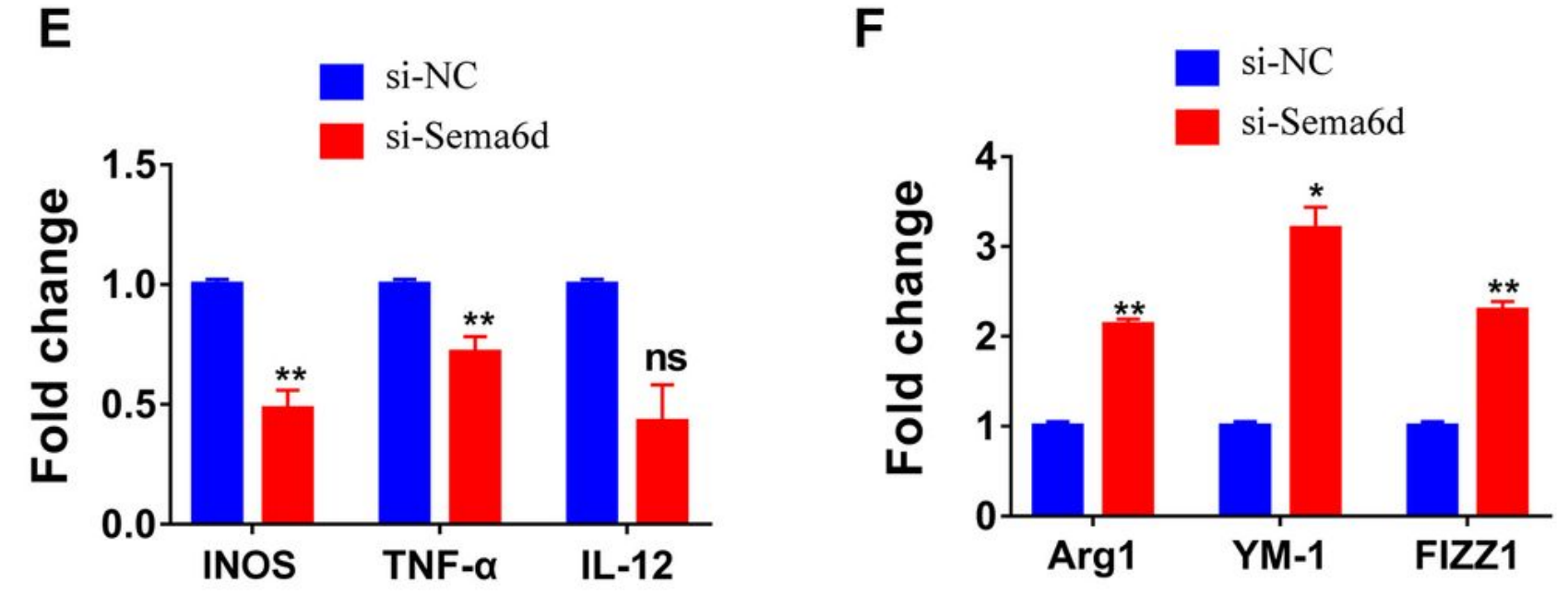

Figure 5

AK141955, miR-124-3p, and sema6d play important roles in macrophage polarization. (A) Primary BMDMs were transfected with NC Smart Silencer or AK141955 Smart Silencer for $48 \mathrm{~h}$. The expression levels of NOS2, TNF-a, and IL-12 were determined using qPCR. (B) Primary BMDMs were transfected with NC Smart Silencer or AK141955 Smart Silencer for $48 \mathrm{~h}$. The expression levels of Arg-1, YM-1, and FIZZ1 were determined using qPCR. (C) Primary BMDMs were transfected with an inhibitor control or miR-124- 
$3 p$ inhibitor for $48 \mathrm{~h}$. The expression levels of NOS2, TNF-a, and IL-12 were determined using qPCR. (D) Primary BMDMs were transfected with an miR-124-3p control or miR-124-3p inhibitor for $48 \mathrm{~h}$. The expression levels of Arg-1, YM-1, and FIZZ1 were determined using qPCR. (E) Primary BMDMs were transfected with NC Smart Silencer or Sema6d Smart Silencer for $48 \mathrm{~h}$. The expression levels of NOS2, TNF- $a$, and IL-12 were determined using APCR. (F) Primary BMDMs were transfected with NC Smart Silencer or Sema6d Smart Silencer for $48 \mathrm{~h}$. The expression levels of Arg-1, YM-1, and FIZZ1 were determined using qPCR. Data are reported as mean \pm s.d. of replicate analyses. ${ }^{*} p<0.05,{ }^{\star} * p<0.01$, ns indicates no significance based on Student's t-test.

A

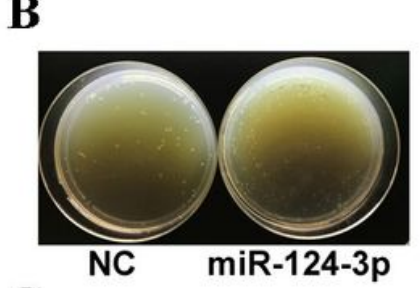

$\mathrm{C}$
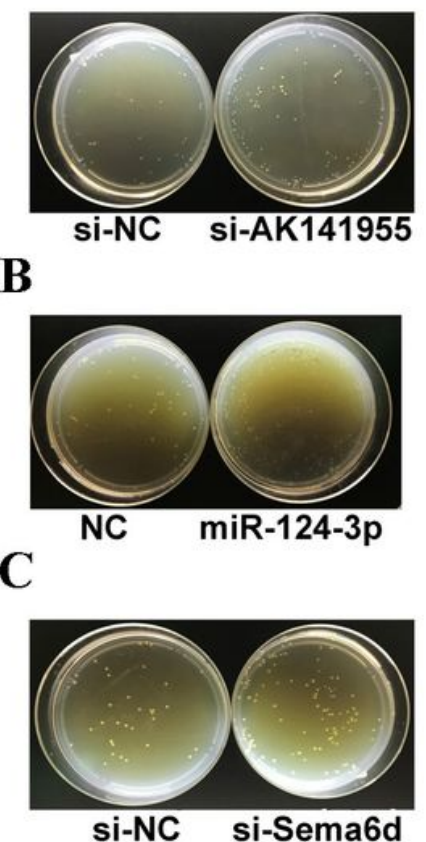
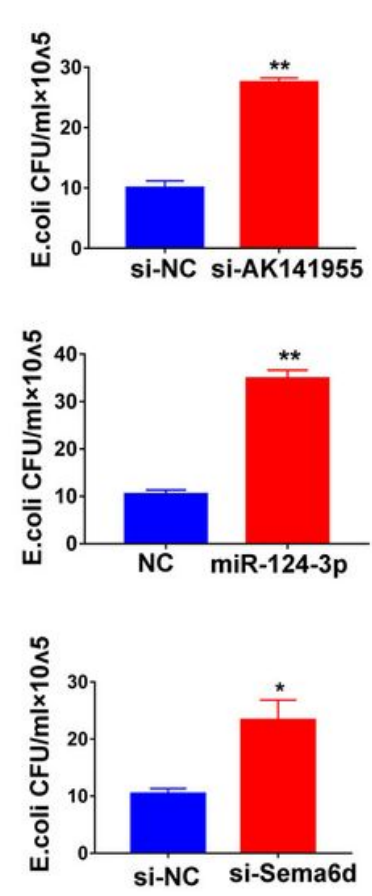

D

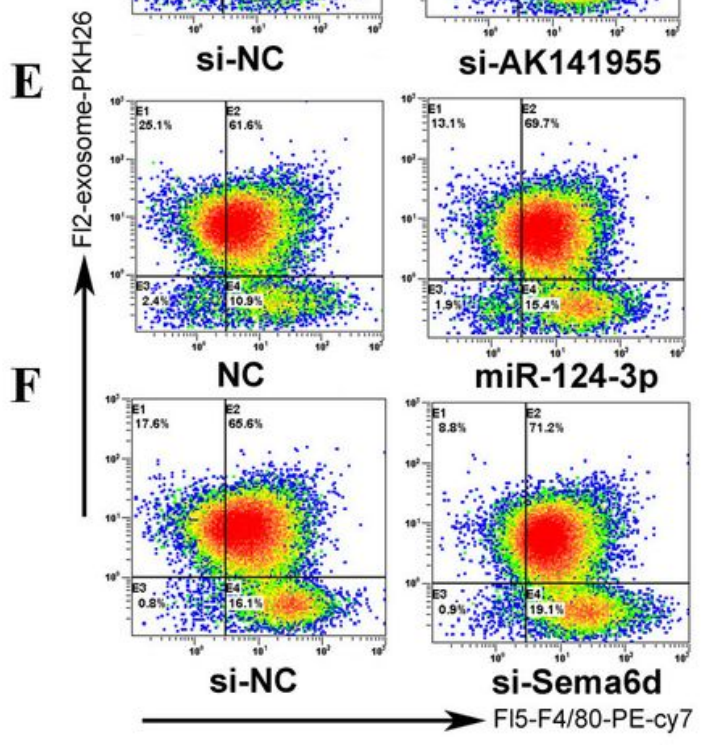

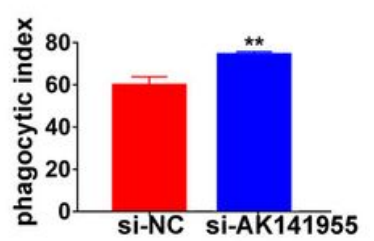
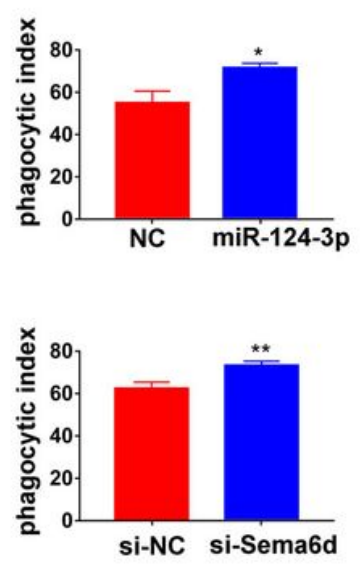

\section{Figure 6}

AK141955, miR-124-3p, and Sema6d regulate macrophage functions associated with the M1 and M2 phenotypes. (A) BMDMs were transfected with NC Smart Silencer or AK141955 Smart Silencer to observe the bactericidal activity of IncRNA 141955. (B) BMDMs were transfected with NC or miR-124-3p mimic to observe the bactericidal activity of miR-124-3p. (C) BMDMs were transfected with NC Smart Silencer or Sema6d Smart Silencer to observe the bactericidal activity of Sema6d. (D) Primary BMDMs were transfected with NC Smart Silencer or AK141955 Smart Silencer for 48 h. After transfection, phagocytosis assays were performed. (E) Primary BMDMs were transfected with NC or an miR-124-3p mimic for $48 \mathrm{~h}$. After transfection, phagocytosis assays were performed. (F) Primary BMDMs were transfected with NC Smart Silencer or Sema6d Smart Silencer for $48 \mathrm{~h}$. After transfection, phagocytosis assays were performed. Data are reported as mean \pm s.d. of replicate analyses. ${ }^{*} p<0.05,{ }^{\star *} p<0.01$ based on Student's t-tests. 
A

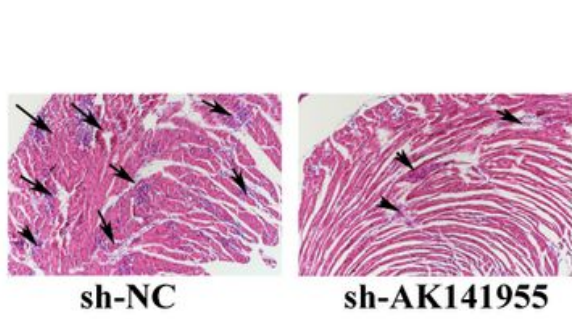

$\mathbf{E}$

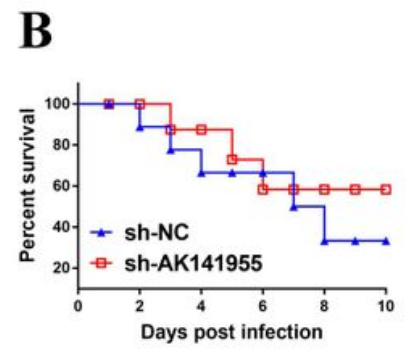

C

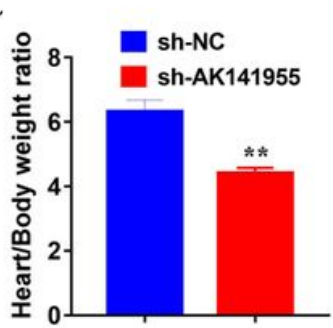

D

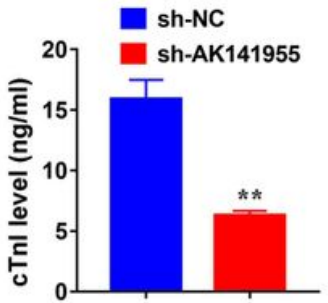

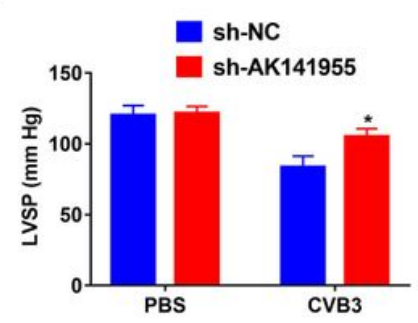
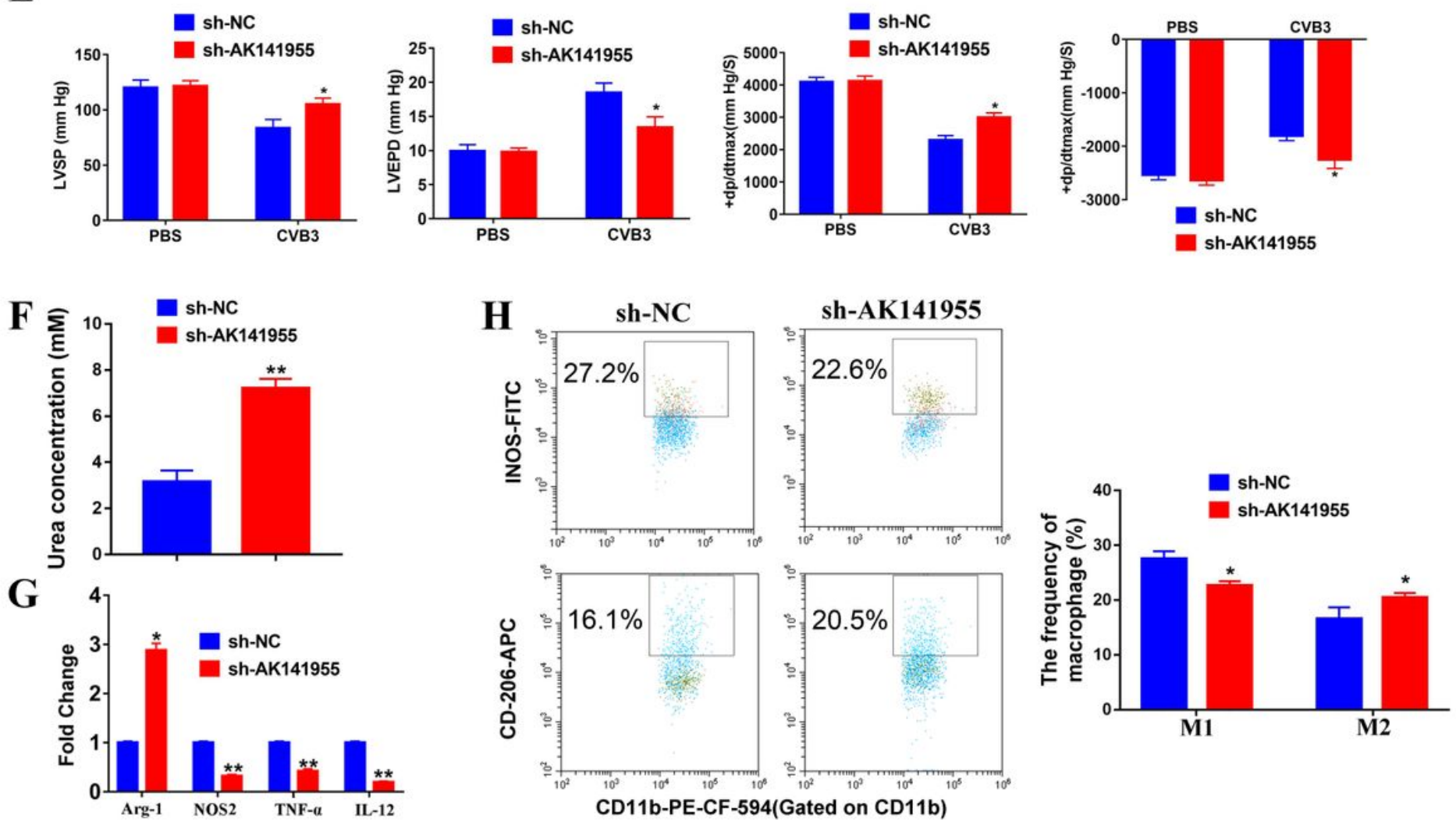

G

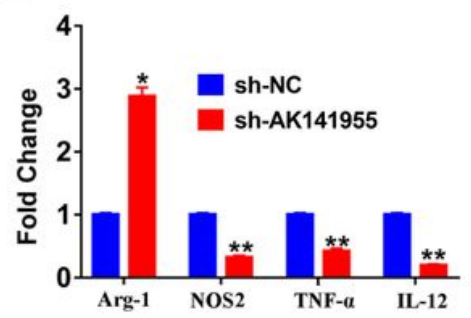

CD11b-PE-CF-594(Gated on CD11b)

\section{Figure 7}

AK141955 attenuated myocardial inflammation during CVB3-induced VM. Adeno-associated viruses from the AK141955 knockdown and control groups were injected into mice through the caudal vein. After the injection, both groups of mice were intraperitoneally injected with CVB3. (A) On day 7 after CVB3 injection, H\&E staining was performed on heart sections of both groups (Scale bar $=100 \mu \mathrm{m})$. (B) The survival rate of mice was observed until day 10 post-infection. (C-D) VM parameters including heart/bodyweight ratio (C) and cTnl levels (D) on day 7 post-infection were evaluated. (E) The left ventricular (LV) performance was assessed using a self-made polyethylene catheter inserted via the right carotid artery. The pressure signal of the left ventricle was recorded continuously and stored in a computer to determine off-line LV end-diastolic pressure (LVEDP), LV systolic pressure (LVSP), maximum first derivative of $L V$ pressure (dP/dtmax), and minimum first derivative of $L V$ pressure $(-\mathrm{dP} / \mathrm{dtmax})$. (F) Tissue homogenates of myocardial-infiltrating macrophages in sh-NC and sh-AK141955 mice during CVB3-induced VM were prepared. Arginase activity of macrophages was assessed by an assay of urea production from arginine substrate and was normalized to cell counts. (G) Phenotypic analysis of 
myocardial-infiltrating macrophages in sh-NC and sh-AK141955 mice during CVB3-induced VM. NOS2, Arg1, FIZZ1, and YM-1 of sorted F4/80+ macrophages were determined by qPCR. (H) Myocardialinfiltrating leucocytes were isolated from the hearts after enzymatic digestion. The percentages of iNOSFITC+ or CD-206-APC cells were analyzed by FACS. Data are reported as mean \pm s.d. of replicate analyses. ${ }^{*} p<0.05,{ }^{* \star} p<0.01$ based on Student's t-tests.

\section{Supplementary Files}

This is a list of supplementary files associated with this preprint. Click to download.

- Additionalfile1.docx

- Additionalfile2.xlsx

- Additionalfile3.xlsx

- Peerreviewers.docx

- authorinformation.docx 